\title{
Sólidos de revolución con GeoGebra
}

\author{
| Revolution solids with GeoGebra | \\ D José Luis Vergara Ibarra \\ ingjosevergaraibarra@gmail.com \\ Colegio 23 de Octubre \\ Montecristi - Ecuador
}

Resumen: Este artículo muestra cómo diseñar sólidos de revolución con el software GeoGebra. Primero en cada sección se dan las definiciones pertinentes para este propósito, luego de esto se realiza la parametrización de las curvas en $\mathbb{R}^{3}$ y de la superficie acotada por estas curvas, finalmente mediante comandos y deslizadores se obtiene el sólido de revolución. Los sólidos obtenidos son dinámicos y se pueden representar en diferentes perspectivas.

Palabras Clave: sólido de revolución, parametrización, curva, superficie.

Abstract: This paper shows how to design revolution solids with GeoGebra software. First in each section the relevant definitions are given for this purpose, after this the curves are parameterized at $\mathbb{R}^{3}$ and the surface bounded by these curves, finally by commands and sliders the solid of revolution is obtained. The solids obtained are dynamic and can be represented in different perspectives.

Keywords: solid of revolution, parameterization, curve, surface.

\section{Introducción}

Los sólidos de revolución son ampliamente estudiados ya que tienen importantes aplicaciones en muchas ramas de la ingeniería. Algunos de estos ejemplos son: ejes, embudos, píldoras, botellas, pistones, frenos de tambor, entre otros (Bruce y Larson, 2010, p.458). Tradicionalmente estos sólidos de revolución son generados por el rastro de una curva cerrada (Del Río, 2016 y León, 2018). También se elaboran sólidos de revolución mediante una secuencia de curvas cerradas o polígonos (Carrazedo y Vieira, 2017).

Una de las dificultades que presentan los libros (cálculo en una variable) Bruce y Larson (2010), Stewart (2018), Smith y Minton (2012), Zill y Wright (2011) es abordar este tema con sólidos de revolución estáticos y con pocos ejemplos donde no se conoce el objeto tridimensional que se está midiendo.

En este sentido, el objetivo principal de este trabajo es poder crear y ver de manera dinámica el desarrollo del sólido desde que la superficie a rotar está en dos dimensiones hasta completar el sólido en tres dimensiones. En cuanto a la forma, es importante mencionar lo siguiente: el sólido de revolución obtenido no tiene apariencia hueca en su interior; pues, en este caso, lo que se visualizará es una superficie de revolución y esto puede ocasionar confusión de estos dos conceptos; en la frontera del sólido no existen grietas o huecos. Tomando en cuenta estos aspectos y gracias a que se pueden cambiar las 
propiedades de forma simultánea e independiente de los objetos que constituyen la estructura del sólido se obtendrá un estilo real y personalizado. Una forma de parametrización de curvas y superficies mediante GeoGebra son abordadas por (Mora F, 2018, pp. 2-13).

La construcción de sólidos de revolución se hará mediante parametrizaciones de curvas y superficies que serán rotadas alrededor de un eje en particular. La parametrización de curvas, superficies y la obtención de las superficies de revolución a partir de las curvas proporciona un método distinto de obtener sólidos de revolución.

\section{Parametrización de curvas}

Tapp (2015) afirma que si $x(t), y(t)$ y $z(t)$ son las coordenadas en el tiempo $t$ de un objeto moviéndose en el espacio. El vector de posición de un objeto en cualquier tiempo $t$ es el vector

$$
\gamma(t)=(x(t), y(t), z(t))
$$

Una función suave $\gamma$ significa que cada una de las tres funciones componentes, $x(t), y(t)$ y $z(t)$, por separado es suave en el sentido de que se puede diferenciar $k$ veces.

Definición 1 (Tapp, 2015)

Una parametrización de una curva en $\mathbb{R}^{3}$ es una función suave $\gamma: \mathrm{I} \longrightarrow \mathbb{R}^{3}$, donde $\mathrm{I} \subset \mathbb{R}$ es un intervalo.

Lema 1 (Tapp, 2015) Si I es un intervalo y $\gamma: I \longrightarrow \mathbb{R}$, es una función suave, entonces $\gamma(t)=(t, f(t))$ modela un objeto que atraviesa la gráfica de $y=f(x)$.

Bajo las hipótesis del lema 1, la curva en el plano XY del espacio $\mathbb{R}^{3}$ tiene la forma $\gamma(t)=(t, f(t), 0)$.

\subsection{Parametrización de rectas y segmentos en el espacio}

Si la recta L pasa por P en dirección de $v$ (Mora W, 2019) entonces una parametrización es

$$
L: r(t)=P+t v, t \in \mathbb{R}
$$

$\nu$ es el vector director. EL segmento que inicia en A y termina en B se puede parametrizar como

$$
C: r(t)=A+t(B-A), \quad t \in[0,1]
$$

\subsection{Parametrización de curvas en el espacio con GeoGebra}

Para este propósito se escribe en la entrada Curva( $<$ Expresión $>,<$ Expresión $>,<$ Expresión $>,<$ Parámetro $>,<$ Valor inicial $>$, $<$ Valor final $>$ ), donde en los primeros tres argumentos se escriben $x(t)$, $y(t)$ y $z(t)$, respectivamente, en el cuarto argumento se escribe la variable $t$ y en los dos últimos argumentos los extremos del intervalo donde $t$ varía.

\section{Ejemplo 1}

La función $f(x)=-x^{2}-2$ que va desde el punto $(-2,-6)$ al punto $(2,-6)$, se parametriza de la siguiente manera: Se define $x=t$ y así $f(t)=-t^{2}-2$ para $t \in[-2,2]$, entonces la curva en $\mathbb{R}^{3}$ es $C:(t, f(t), 0)=\left(t,-t^{2}-2,0\right), t \in[-2,2]$

En GeoGebra se escribe $f(x)$ (expresión del lado derecho) en la entrada y luego se escribe en el comando Curva $(t, f(t), 0, t,-2,2)$ así se obtiene en la vista algebraica una ecuación paramétrica 
de la forma

$$
\left.C: \begin{array}{l}
x(t)=t \\
y(t)=-t^{2}-2 \\
z(t)=0
\end{array}\right\}, \quad-2 \leqslant t \leqslant 2
$$

y en la vista gráfica 3D la curva deseada. Esto se muestra en la Figura 1

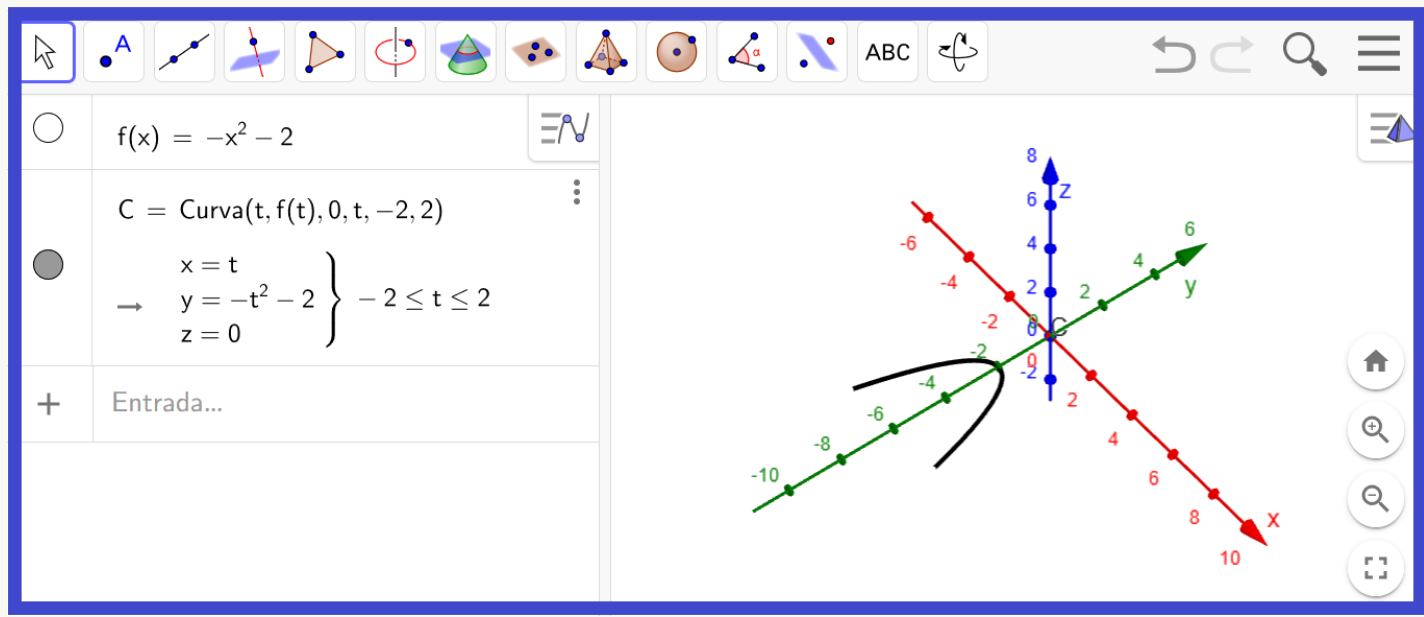

Figura 1: Curva C.

\section{Ejemplo 2}

Las parametrizaciones de las curvas bajo las funciones $f(x)=x^{2}, g(x)=2-x$ y $h(x)=0$ que encierran una área en particular se obtienen de la siguiente forma:

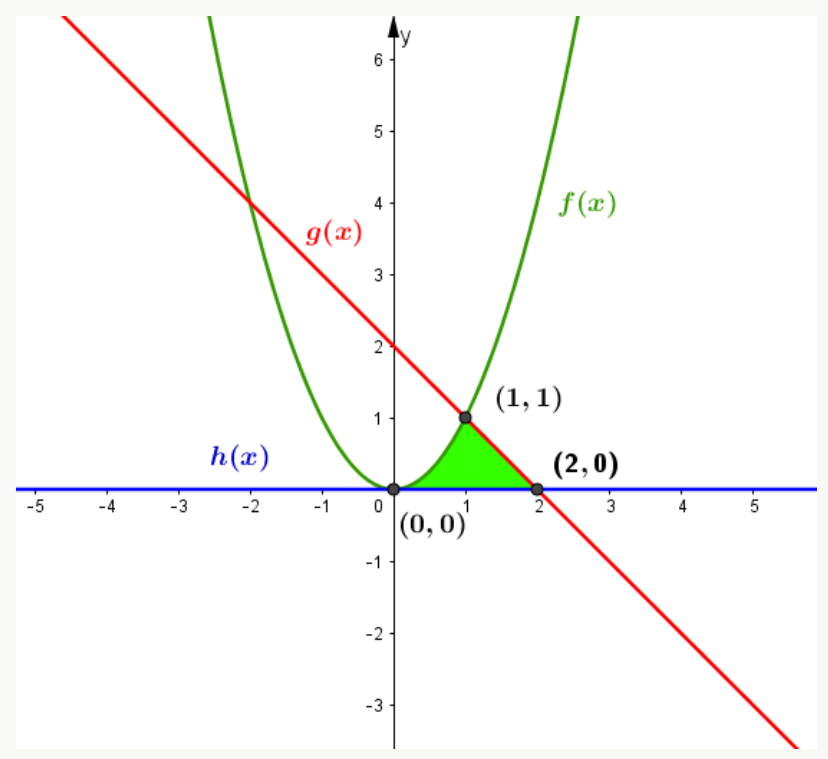

Figura 2: Puntos de intersección de las funciones.

Los puntos de intersección se obtienen haciendo operaciones algebraicas básicas, o por medio de la vista gráfica 2D como se muestra en la Figura 2. Estos puntos son $(0,0),(1,1)$ y $(2,0), f(x)$ desde $(0,0)$ a $(1,1), g(x)$ desde $(1,1)$ al $(2,0)$ y $h(x)$ del $(0,0)$ hasta $(2,0)$. 
Así se obtienen las curvas,

$$
\begin{aligned}
& \left.\begin{array}{rl}
x(t) & =t \\
C_{1}: & y(t)=t^{2} \\
z(t) & =0
\end{array}\right\}, \quad 0 \leqslant t \leqslant 1 \\
& \left.\begin{array}{rl}
x(t) & =t \\
C_{2}: & y(t)=2-t \\
z(t) & =0
\end{array}\right\}, \quad 1 \leqslant t \leqslant 2 \\
& \left.\begin{array}{c}
x(t)=t \\
C_{3}: \begin{array}{l}
y(t) \\
z(t)
\end{array}=0
\end{array}\right\}, \quad 0 \leqslant t \leqslant 2
\end{aligned}
$$

para $f(x), g(x)$ y $h(x)$ respectivamente.

En la Figura 3 se ilustra la construcción de estas curvas, para ello se sigue el proceso del Ejemplo 1 para cada curva.

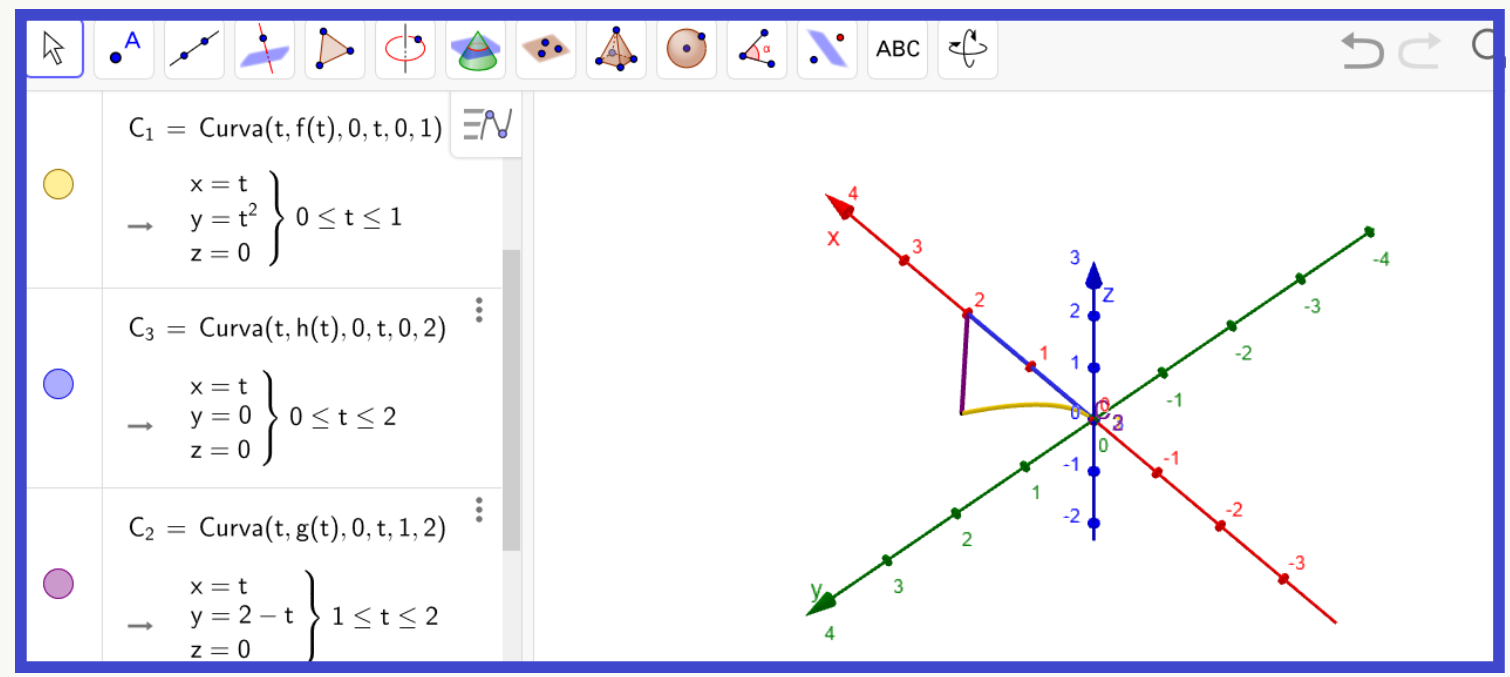

Figura 3: $C_{1}, C_{2}$ y $C_{3}$.

\section{Parametrización de superficies}

Existe una conexión entre las funciones de valores vectoriales y las ecuaciones paramétricas. En esta sección se amplía la noción de curva en el espacio.

Definición 2 (Tapp, 2015)

Una parametrización de una superficie en $\mathbb{R}^{3}$ es una función suave $\sigma: U \longrightarrow \mathbb{R}^{3}$, donde $\mathrm{U} \subset \mathbb{R}^{2}$. 


\subsection{Superficie Reglada}

Definición 3 (Tapp, 2015)

Una superficie parametrizada reglada es una superficie parametrizada $\sigma: U \subset \mathbb{R}^{2} \longrightarrow \mathbb{R}^{3}$ que tiene la forma $\sigma(s, t)=\gamma(s)+t w(s)$, donde $\gamma$ y $w$ son curvas en el espacio.

\subsection{Parametrización de superficies acotadas por curvas con GeoGebra}

Suponga que $\gamma_{1}(t)$ y $\gamma_{2}(t)$ son dos curvas que satisfacen la definición 1, entonces la parametrización de la superficie encerrada por estas curvas tiene la forma $S(s, t)=s \gamma_{1}+(1-s) \gamma_{2}$ (ver definición 3 ), donde $0 \leqslant s \leqslant 1$ y $a \leqslant t \leqslant b$, con $a, b \in \mathbb{R}$. Este hecho se usa para obtener la superficie y la parametrización con GeoGebra, es decir, se utiliza el siguiente comando

Superficie $\left(s \gamma_{1}+(1-s) \gamma_{2}, t, a, b, s, 0,1\right)$.

\section{Ejemplo 3}

La parametrización de las curvas bajo las funciones $f(x)=-x^{2}+1$ y $g(x)=x^{2}-1$ se obtiene calculando los puntos de intersección y a partir de esta información se determina el intervalo de variación de t para cada curva (ver Ejemplo 2). Estas curvas encierran una área que es parametrizada teniendo las curvas de cada función, escribimos estos datos en la entrada de GeoGebra, es decir, Superficie $(\mathbf{s} \mathbf{a}(\mathbf{t})+(\mathbf{1}-\mathbf{s}) \mathbf{b}(\mathbf{t}), \mathbf{t}, \mathbf{- 1}, \mathbf{1}, \mathbf{s}, \mathbf{0}, \mathbf{1})$, así se obtiene en la Figura 4 el resultado deseado.

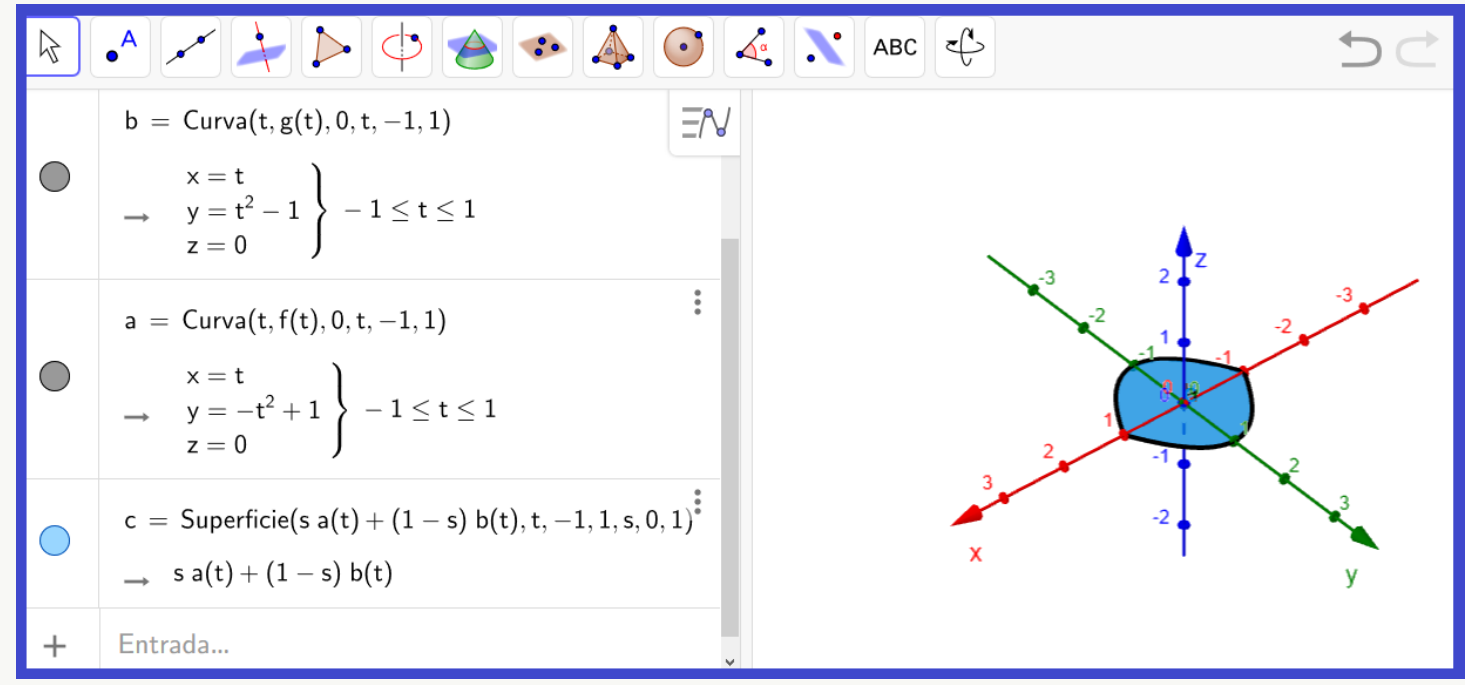

Figura 4: Superficie c.

\section{Ejemplo 4}

En este ejemplo se construye la parametrización de la superficie acotada en el primer cuadrante por dos funciones y una recta $y=4-x^{2}, y=1$ y $x=0$. Mediante un cálculo directo se tiene que los puntos de intersección son $(0,4),(0,1)$ y $(\sqrt{3}, 1)$. Las parametrizaciones de las curvas son

$$
\left.b: \begin{array}{l}
x(t)=t \\
y(t)=4-t^{2} \\
z(t)=0
\end{array}\right\}, \quad 0 \leqslant t \leqslant \sqrt{3}
$$




$$
\begin{aligned}
& \left.c: \begin{array}{l}
x(t)=t \\
y(t)=1 \\
z(t)=0
\end{array}\right\}, \quad 0 \leqslant t \leqslant \sqrt{3}
\end{aligned}
$$

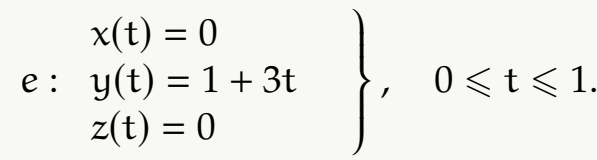

La parametrización de la superficie encerrada por las curvas es

$$
d=s c(t)+(1-s) b(t), \text { para } 0 \leqslant t \leqslant \sqrt{3} \text { y } 0 \leqslant s \leqslant 1
$$

Para la construcción de la superficie se escribe la expresión Superficie $(\mathbf{s} \mathbf{c}(\mathbf{t})+(1-$ s) $b(t), t, 0, \sqrt{3}, \mathbf{s}, \mathbf{0}, \mathbf{1})$, en la entrada de GeoGebra y se obtiene la siguiente ilustración (Figura $5)$.

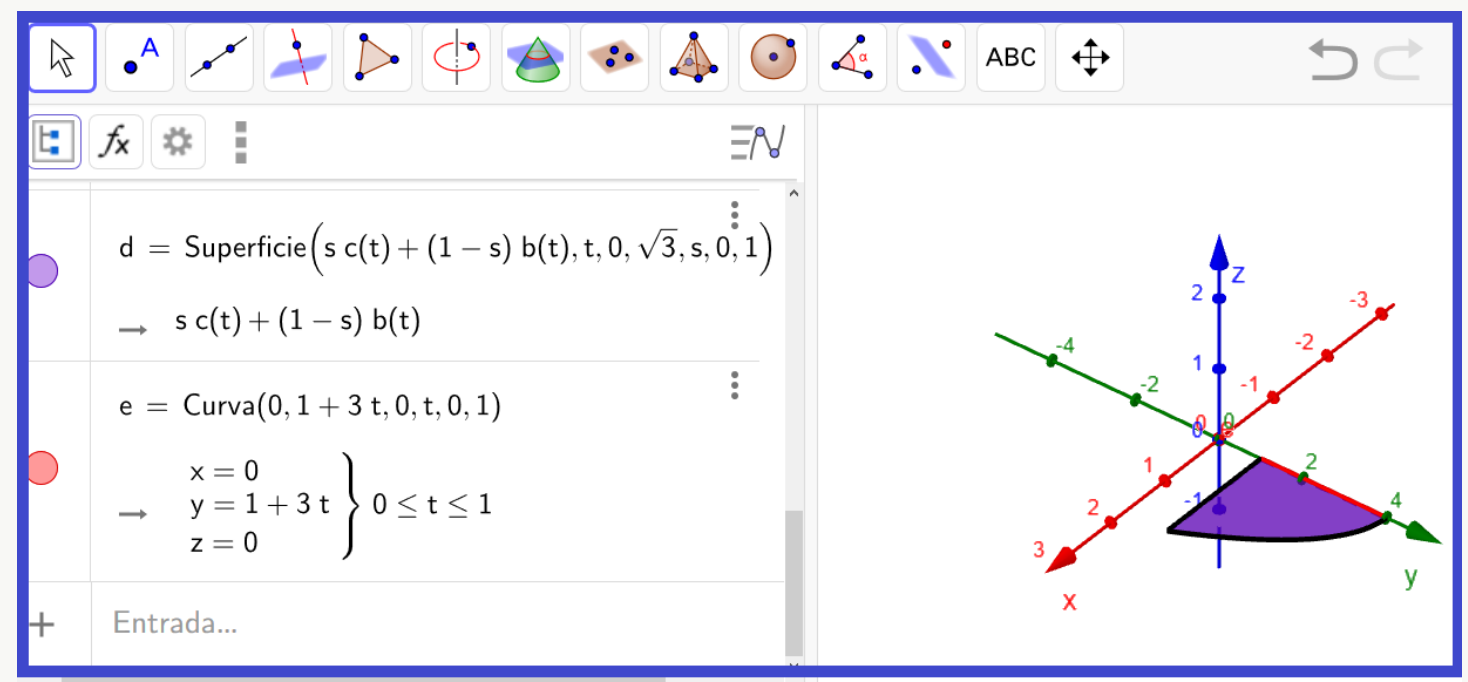

Figura 5: Superficie d.

La ecuación paramétrica (9) que corresponde al segmento marcado en rojo de la Figura 5 es obtenida utilizando la parametrización de un segmento, ver ecuación (2).

En ocasiones dependiendo de la forma que tienen las curvas que acotan a una superficie en particular se tendrá que hacer dos parametrizaciones de la superficie. En el siguiente ejemplo se realiza esta construcción.

\section{Ejemplo 5}

La superficie acotada por las curvas del Ejemplo 2 tiene dos parametrizaciones,

$$
\mathrm{S}_{1}=s \mathrm{C}_{1}(\mathrm{t})+(1-\mathrm{s}) \mathrm{C}_{3}(\mathrm{t}) \text {, para } 0 \leqslant \mathrm{t} \leqslant 1 \text { y } 0 \leqslant s \leqslant 1
$$

y

$$
\mathrm{S}_{2}=s \mathrm{C}_{2}(\mathrm{t})+(1-\mathrm{s}) \mathrm{C}_{3}(\mathrm{t}) \text {, para } 1 \leqslant \mathrm{t} \leqslant 2 \text { y } 0 \leqslant s \leqslant 1
$$

Estas dos parametrizaciones para la superficie se obtienen a partir de las parametrizaciones de las curvas del Ejemplo 2, se sustituyen en (7) adecuadamente y luego se escriben estos datos en la barra de entrada de GeoGebra, obteniendo la siguiente construcción (Figura 6): 


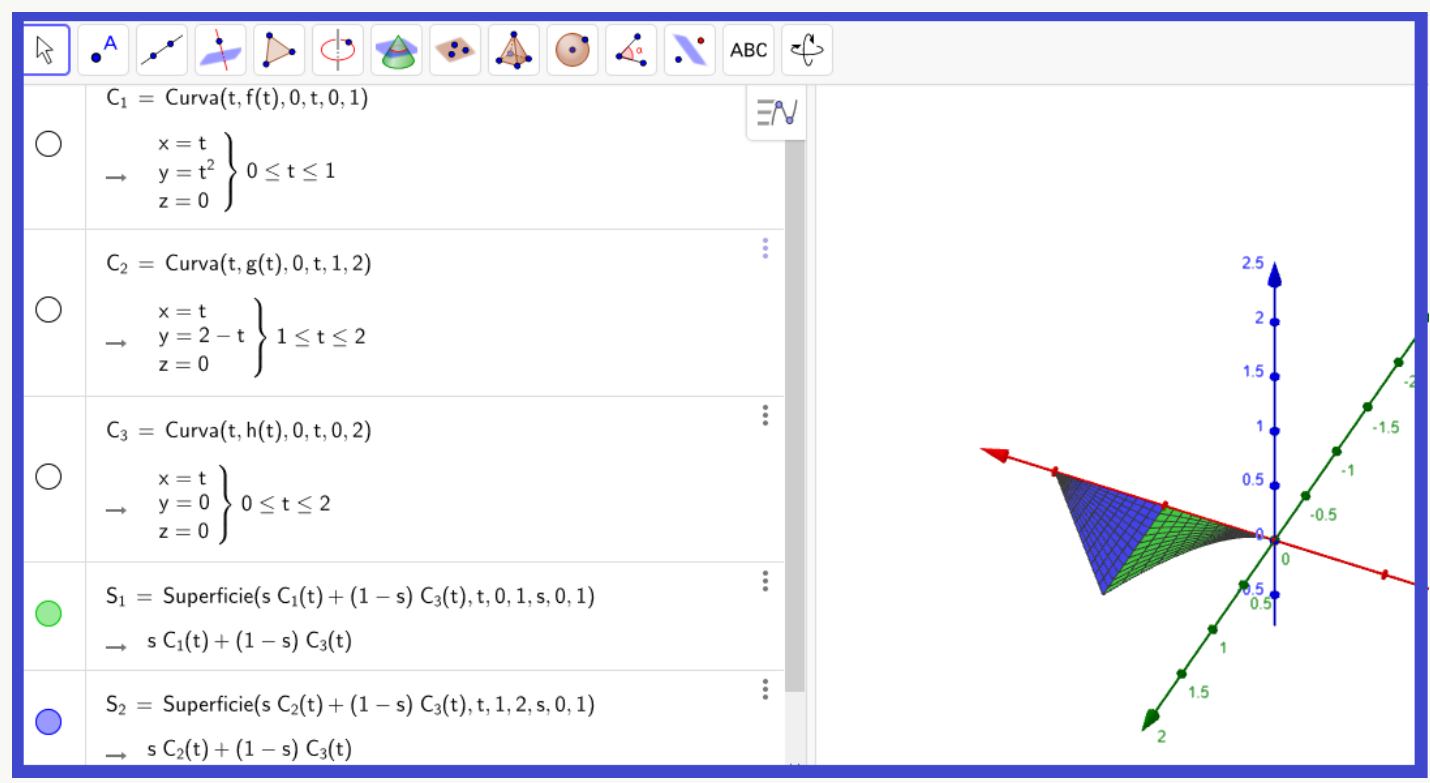

Figura 6: Superficies $S_{1}$ y $S_{2}$.

Modificando las propiedades de las curvas y de las superficies se obtiene la Figura 7, que corresponde al acabado final.

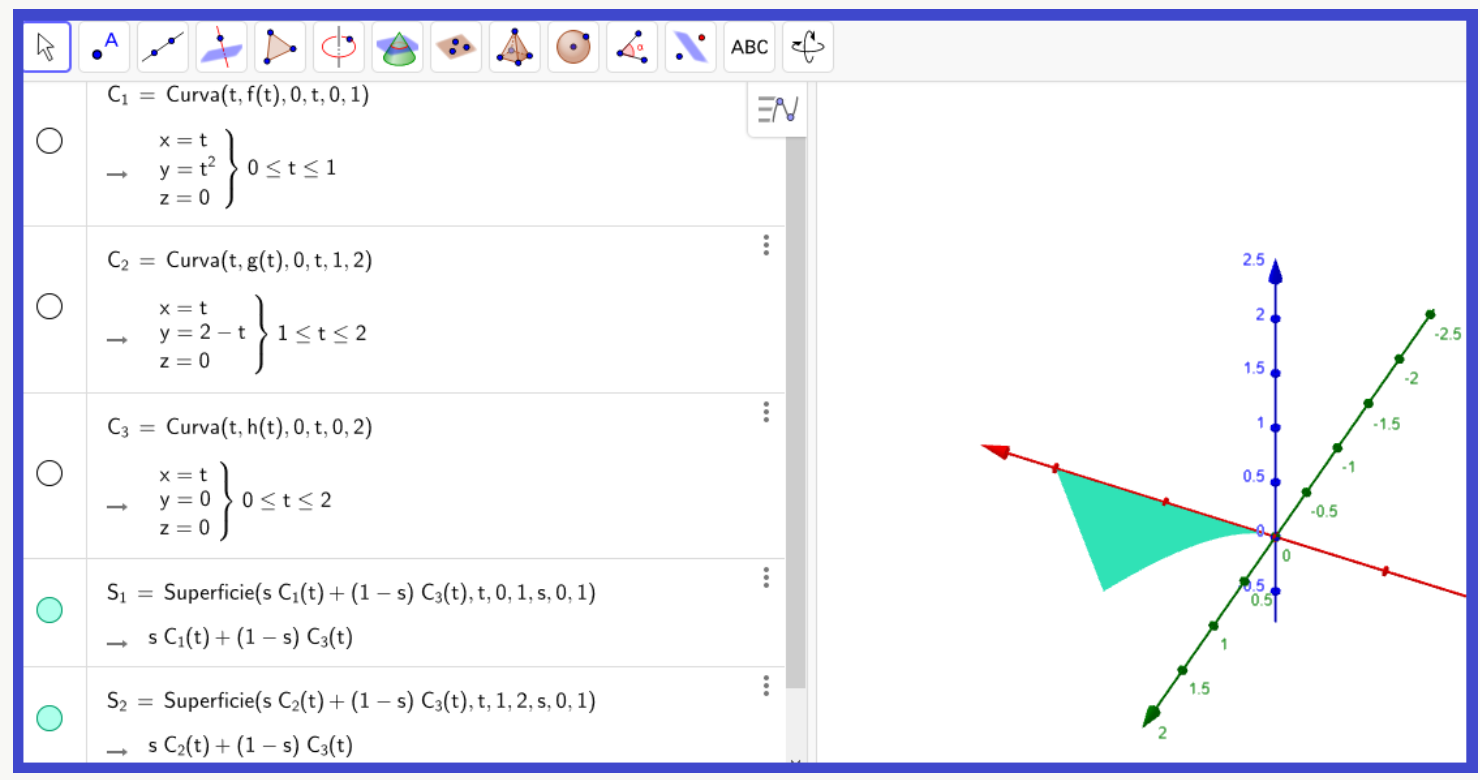

Figura 7: Acabado final

\section{Sólidos de Revolución}

Una región $S \subset \mathbb{R}^{2}$ que es girada alrededor de un eje $L$, genera un sólido $R \subset \mathbb{R}^{3}$ llamado sólido de revolución (Figura 8). Existen algunos métodos para determinar el volumen de un sólido de revolución, por ejemplo el método del disco, arandela, entre otros.

\subsection{Superficies de Revolución}

Una superficie de revolución $S_{r} \subset \mathbb{R}^{3}$ se obtiene al girar una curva respecto a un eje L, esta superficie es la frontera de un sólido de revolución ( $\partial R$ ). Representado en la Figura 9 


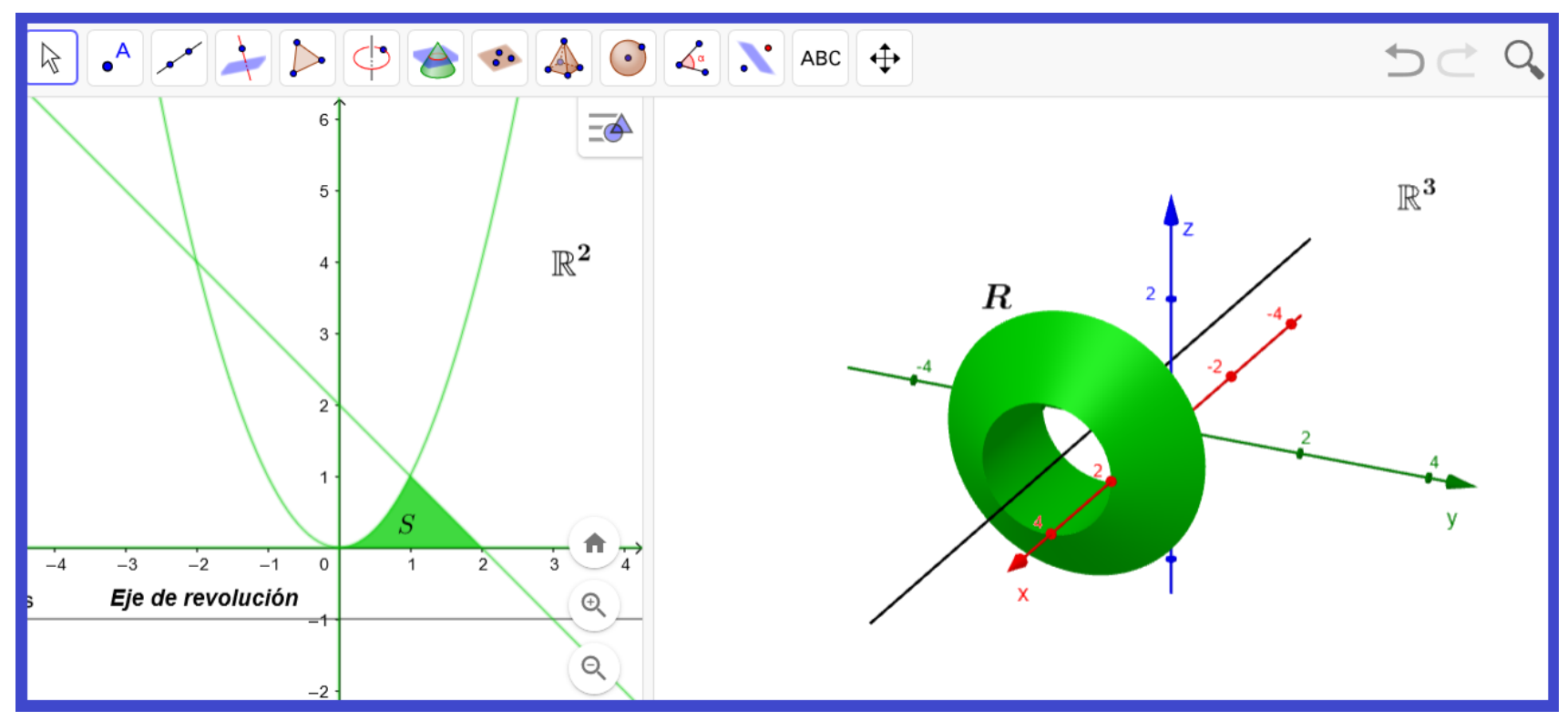

Figura 8: Superficie S y Sólido de revolución R.

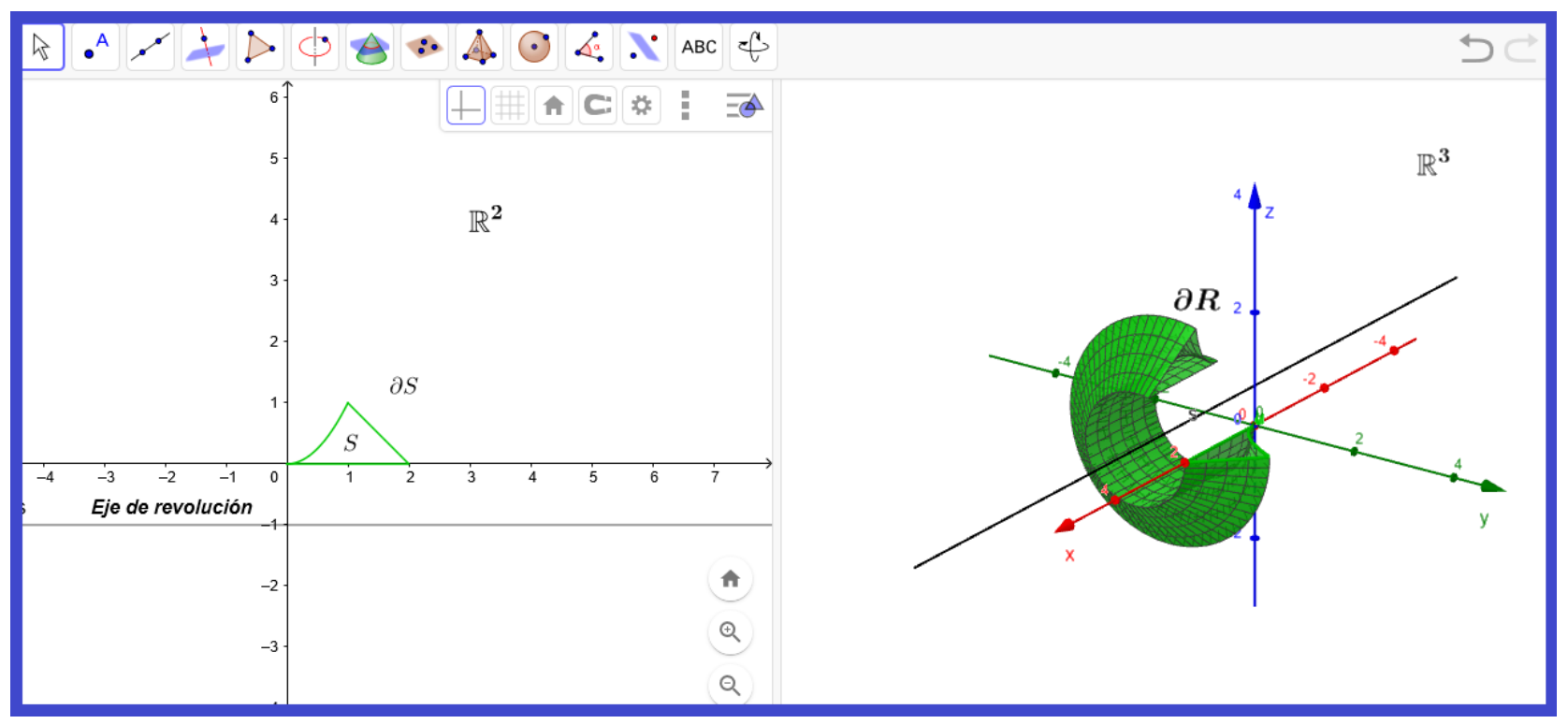

Figura 9: Frontera del sólido $\partial R$.

\section{Construcción de sólidos de revolución}

En esta sección se construirá el sólido de revolución con GeoGebra utilizando la información anterior, además se añade el uso de un deslizador y de dos comandos adicionales los cuales son Superficie( $<$ Curva $>,<$ Ángulo $>,<$ Recta $>$ ) y $\operatorname{Rota}(<$ Objeto $>,<$ Ángulo $>,<$ Eje de rotación $>$ ). El primer comando junto con el deslizador crea una superficie de revolución generada por las curvas y el segundo comando vinculado con el deslizador rota la superficie encerrada por las curvas, así haciendo cambios convenientes en las propiedades de las curvas y la superficie acotada se obtiene el sólido de revolución.

A continuación, los siguientes ejemplos muestran sólidos de revolución dinámicos a través de un único deslizador. 


\section{Ejemplo 6}

Para construir el sólido de revolución formado al girar la región acotada por $f(x)=2-x^{2} y$ $g(x)=1$ alrededor de la recta $y=1$, se sigue el proceso de cualquiera de los ejemplos anteriores, haciendo esto se obtiene la siguiente figura

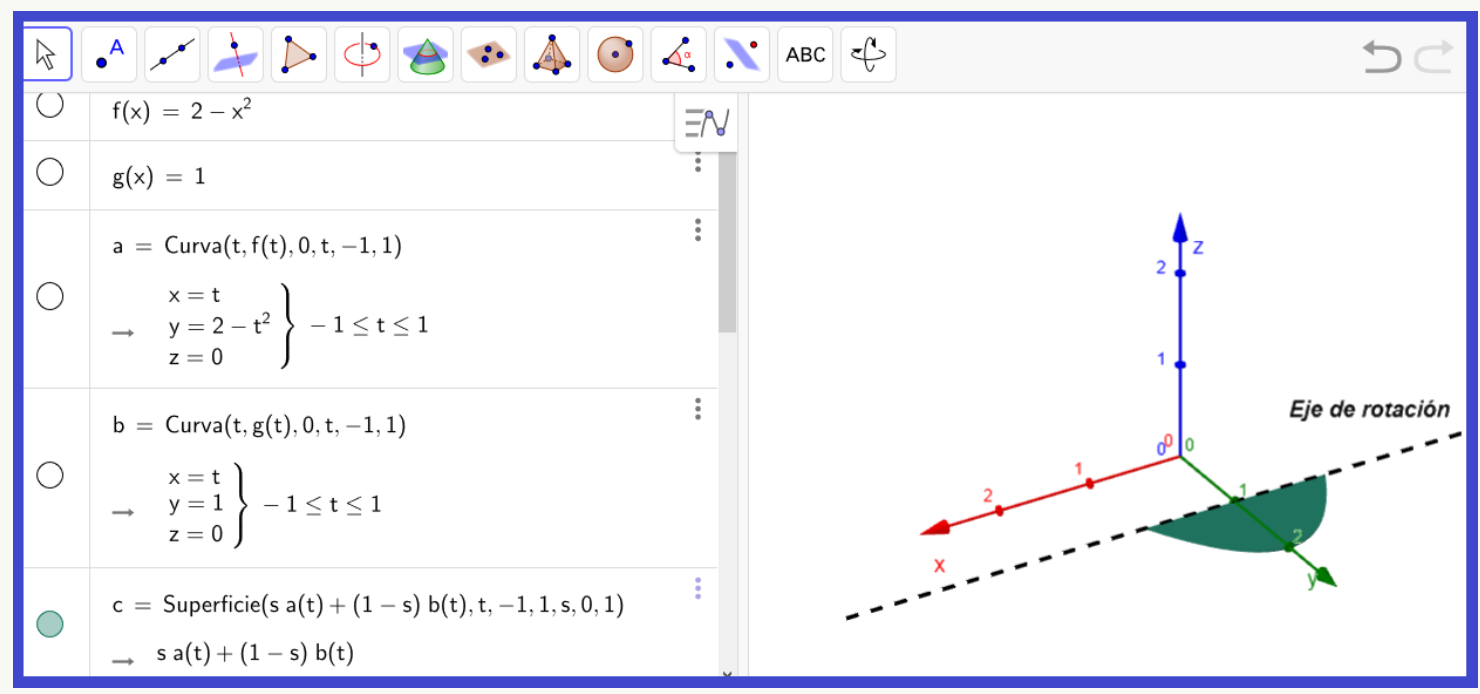

Figura 10: Curvas y superficie acotadas.

Una vez que se obtiene la región de la Figura 10 que está compuesta por dos curvas y la superficie acotada por ellas con las mismas propiedades (color, estilo) se construye un deslizador $\alpha$ que varía de 0 a $2 \pi$, luego de esto se construye una superficie de revolución por cada curva, esto se logra utilizando el comando Superficie ( $<$ Curva $>,<$ Ángulo $>,<$ Recta $>$ ). En el primer argumento sustituimos la curva, en el segundo argumento el ángulo $\alpha$ y en el tercero el eje de rotación de la región. Por último rotamos la superficie con ayuda del comando $\mathbf{R o t a}(<\mathrm{Ob}-$ jeto $>,<$ Ángulo $>,<$ Eje de rotación $>$ ). Las curvas y la superficie de este ejemplo están dadas por las expresiones,

$$
\begin{aligned}
& a: \begin{array}{l}
x(t)=t \\
y(t)=2-t^{2} \\
z(t)
\end{array}=0 \\
& \left.b: \quad \begin{array}{l}
x(t)=t \\
y(t)=1 \\
z(t)=0
\end{array}\right\}, \quad-1 \leqslant t \leqslant 1 \leqslant 1 .
\end{aligned}
$$

$\mathrm{y}$

$$
c=s a(t)+(1-s) b(t), \text { para }-1 \leqslant t \leqslant 1 \text { y } 0 \leqslant s \leqslant 1.1
$$

Con las ecuaciones paramétricas de las curvas y la superficie definidas en la vista algebraica $(14,15$ y 16), se escribe en la entrada $\operatorname{Superficie}(a, \alpha, y=1)$ y $\operatorname{Superficie}(b, \alpha, y=1)$, además para rotar la superficie también se escribe $\operatorname{Rota}(c, \alpha, y=1)$. Finalmente animando el deslizador de 0 a $2 \pi$ se construye el sólido dinámico deseado, como se muestra en la Figura 11 


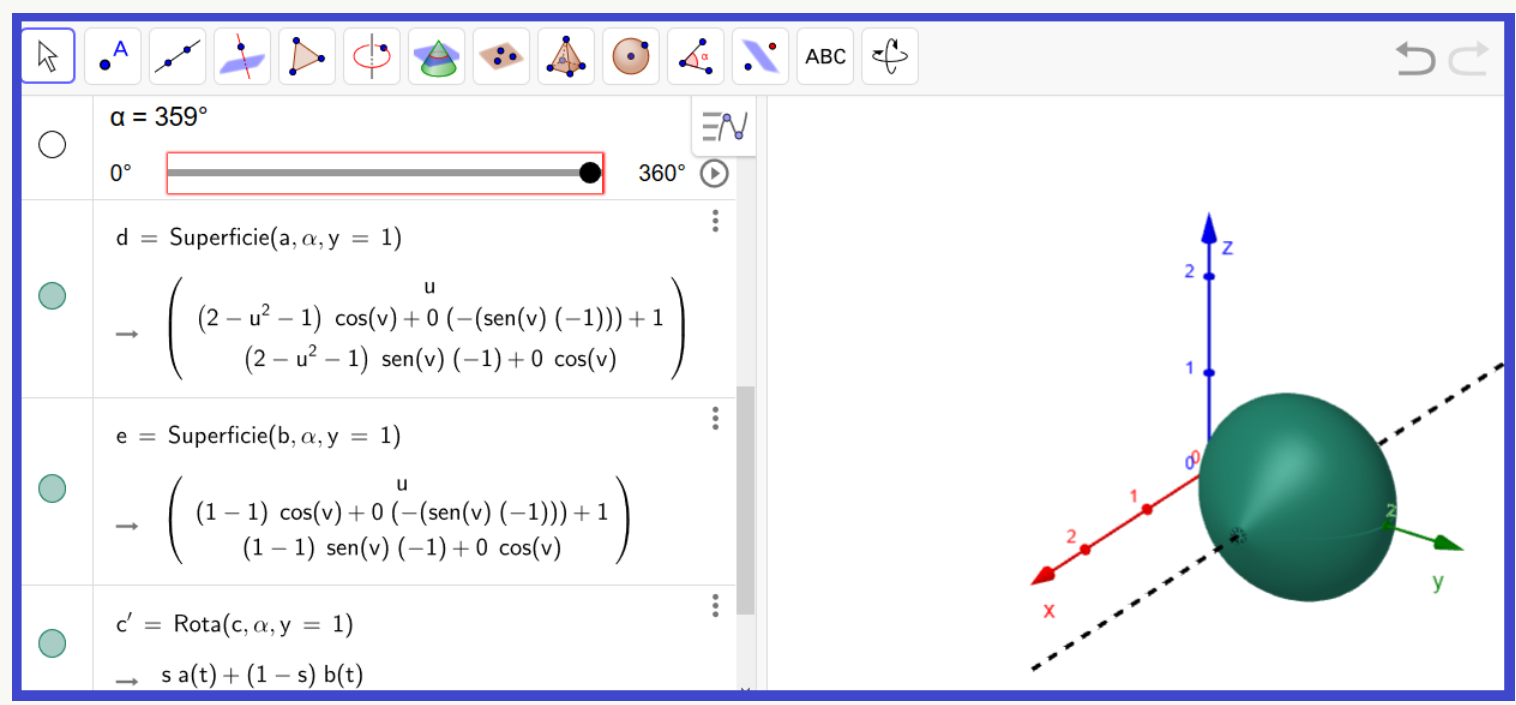

Figura 11: Sólido de revolución 1.

\section{Ejemplo 7}

El sólido formado al girar la región acotada por $h(x)=\sqrt{x}$ y $p(x)=x^{2}$ alrededor del eje $X$ se muestra en la Figura 12:

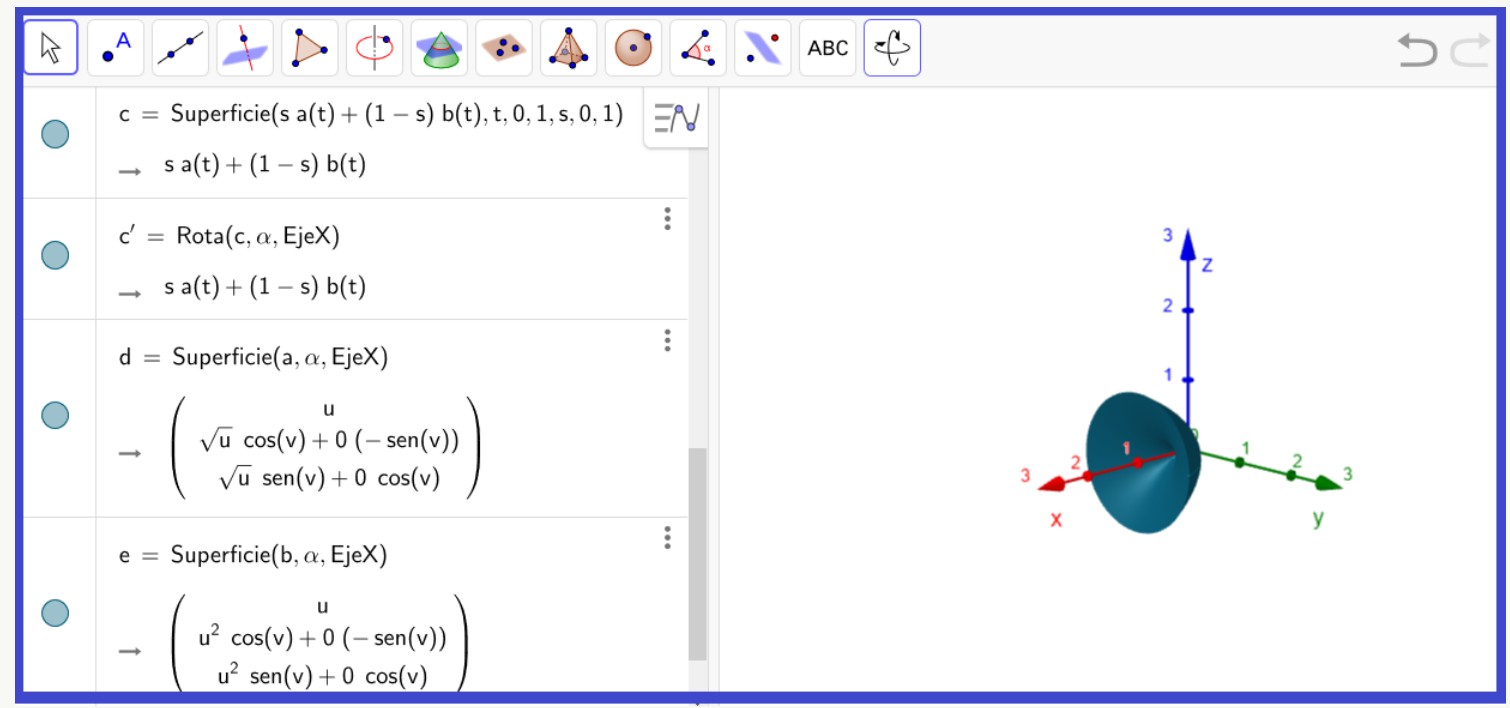

Figura 12: Sólido de revolución 2.

Observe que se sigue el mismo proceso del Ejemplo 6. En la vista algebraica del lado derecho Figura 12 se muestra que el eje de rotación se escribe en los comandos (Rota y Superficie) como EjeX.

Ahora en el Ejemplo 8 se realiza dos variaciones del deslizador $\alpha$ para mostrar el dinamismo del sólido.

\section{Ejemplo 8}

Revolucione la región debajo de la curva $y=\sqrt{x}$ en el intervalo $[0,4]$ sobre el eje $y=-1$.

Según las condiciones del problema las curvas que acotan la superficie a rotar son $y=\sqrt{x}, y=0$ 
$\mathrm{y} x=4$. Por lo que se obtienen las Figuras 13a y $13 \mathrm{~b}$ :

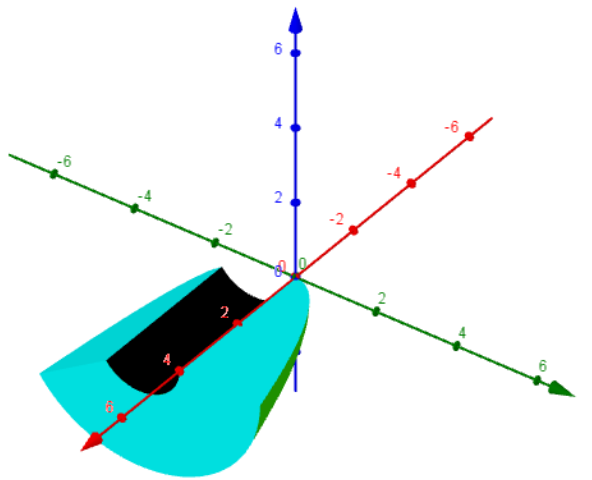

(a) Variación a.

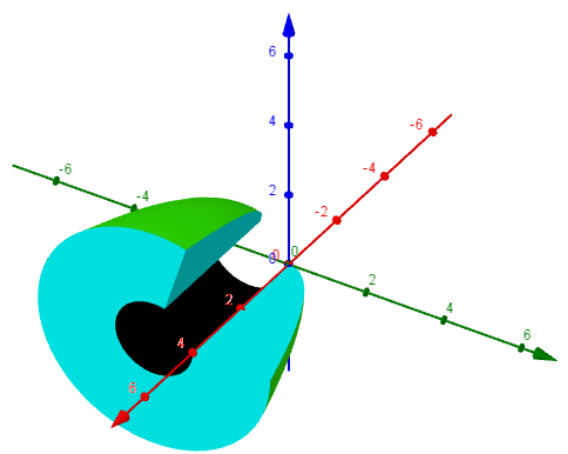

(b) Variación b.

Figura 13: Variaciones

En las Figuras 14 y 15 se muestran dos perspectivas del sólido generado por la superficie acotada por las funciones $f(x)=-(x+2)(x-2)$ y $g(x)=0$ para $-2 \leqslant x \leqslant 2$. El eje de rotación de la superficie es la recta $x=3$.

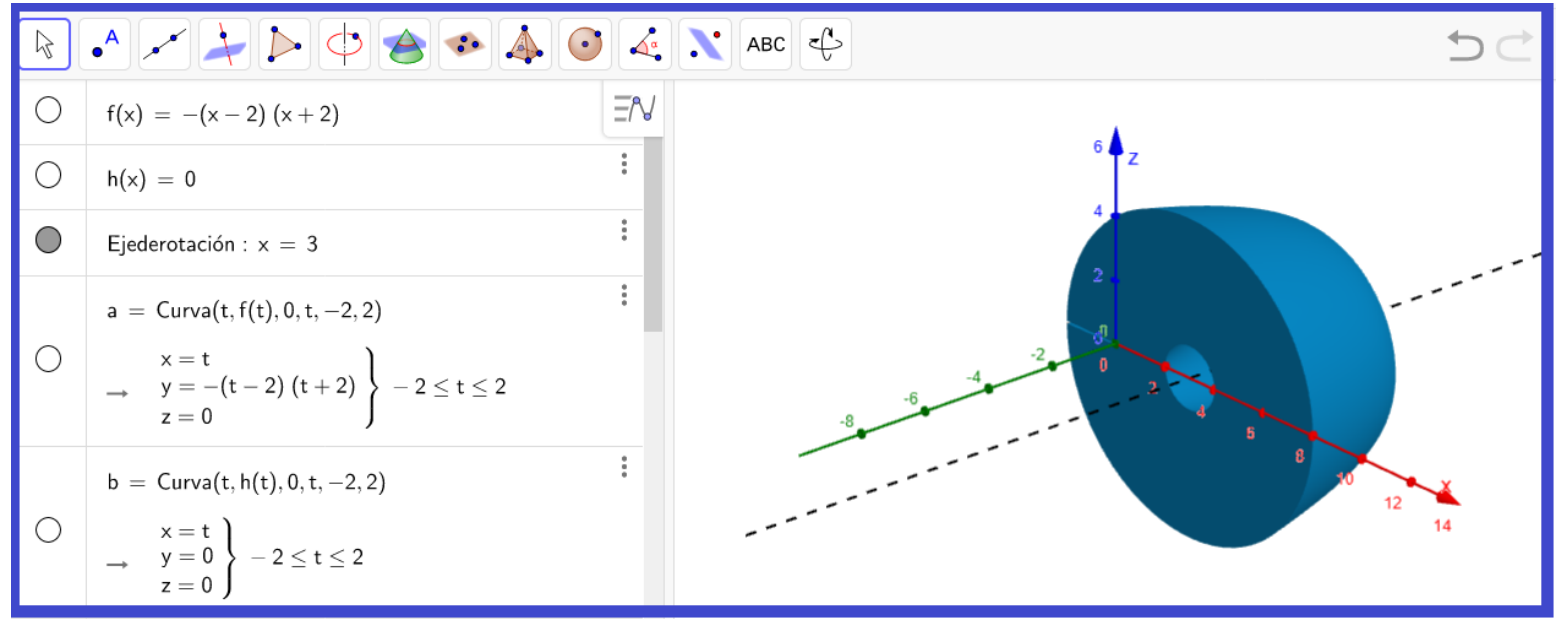

Figura 14: Perspectiva a.

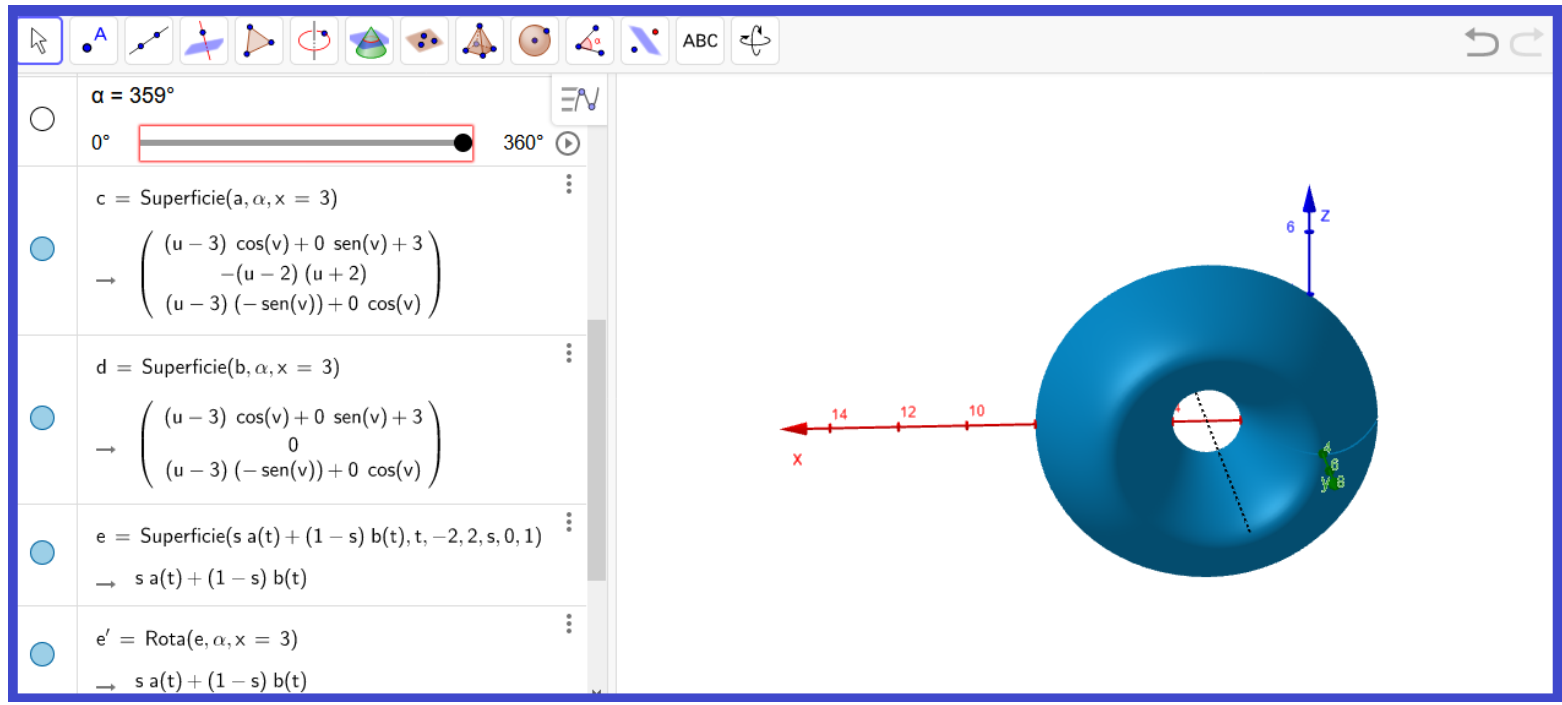

Figura 15: Perspectiva b. 


\section{Conclusión}

Los ejemplos tratados en secuencia para la construcción de sólidos de revolución proporcionan al lector las bases para la construcción de cualquier sólido de revolución e incluso modelar situaciones reales en ciertos campos de la ingeniería. Este estilo de construcción basado en conceptos matemáticos puede servir como apoyo didáctico en la enseñanza-aprendizaje de este tema en sus niveles y áreas pertinentes.

Todo este producto o construcción de sólidos permite inferir algunas propiedades y así ayuda a la interpretación geométrica y analítica, brindando una mejor abstracción para la comprensión objetos tridimensionales.

\section{Referencias}

[1] Bruce, E. y Larson, R. (2010). Cálculo 1 de una variable. México, D.F: McgrawHill/Interamericana.

[2] Del Río, L. (2016). Enseñar y aprender cálculo con ayuda de la vista gráfica 3D de GeoGebra. Revista Digital: Matemática, Educación e Internet, 17(1): 1-13.

[3] León, J., Sánchez, L. y Córdova, R. (2018). Sólidos de revolución haciendo uso de la vista gráfica 3D del GeoGebra. Revista : Acta Latinoamericana de Matemática Educativa, 31(2): 18231827.

[4] Carrazedo, S. y Vieira, C. (2017). Formas de revolução e cálculo de volume. Revista : Centro de Ciências Naturais, 39(1): 142-155. https://doi.org/10.5902/2179460X24428

[5] Stewart, J. (2018). Cálculo. Trascendentes tempranas. México, D.R: Cengage Learning Editores.

[6] Smith, R. \& Minton, R. (2012). Calculus. New York,: The McGraw-Hill Companies.

[7] Zill, D. y Wright, W (2011). Cálculo. Trascendentes tempranas. México, D.F: McgrawHill/Interamericana editores.

[8] Mora, F. (2018). Parametrizaciones de curvas y superficies: Construcción de sólidos con GeoGebra 3D. Revista Digital: Matemática, Educación e Internet 19(1): 1-18.

[9] Tapp, K. (2015). Differential geometry of curves and surfaces. Geometric Methods in Signal and Image Analysis.

[10] Mora, W. (2019). Cálculo en Varias Variables. Visualización interactiva. 2da ed. [ebook] Cartago, Costa Rica. Revista digital, Matemática, Educación e Internet. https://tecdigital.tec.ac.cr/ revistamatematica/Libros/. [Recuperado en Agosto, 2020]. 